\title{
Is the Helium Ion Microscope (Ne) suitable for EBSD sample preparation?
}

\section{Annalena Wolff}

Queensland University of Technology, Brisbane, Queensland, Australia

Focused Ion Beams (FIB) have become increasingly popular for Electron Back Scatter Diffraction (EBSD) sample preparation. The underlying ion-solid interactions of the ion beam with the sample generates a multitude of artefacts in the sample including but not limited to ion implantation, dislocationand vacancy formation as well as amorphization. The FIB-induced artefacts are well recognized for Transmission Electron Microscopy (TEM) lamella preparation and FIB protocols are designed to reduce artefacts, artefacts such as the thickness of amorphous layers of the FIB prepared lamella (1). On the other hand, artefact generation and a thorough understanding on their impact on EBSD measurements is not extensively discussed in literature. Artefact generation which can lead to significant changes in microstructure are now increasingly being reported in literature for FIB EBSD sample polishing $(2,3)$. This work investigates the underlying causes of the artefact formation when using ion beams for EBSD sample preparation and their potential impact on EBSD measurement interpretation. To this end, artefacts that can be induced in $\mathrm{Cu}$ using both Ga FIB and Ne HIM are investigated experimentally for different Ga FIB / Ne HIM polishing parameters using EBSD and TEM. The impact of the underlying ion solid interactions on artefact formation is investigated using Monte Carlo simulations (SRIM). The presented results show how ion beam polishing can induce artefacts that can easily lead to misinterpretation of EBSD results. Ga polishing can induce not only substantial changes in crystal structure but also induce phase transformations even for polishing with lower ion energies at glancing angles. Using high energy (25keV) $\mathrm{Ne}$ ions at glancing angles was found to significantly improve indexing while avoiding sample alterations that could lead to EBSD misinterpretation. The evaluation of SRIM simulation and experimental results suggest that minimizing the number of displacements as well as the resulting ion implantation concentration is essential to avoidance of significant sample alterations during FIB polishing. The results show that Ne HIM produced better results for EBSD sample polishing than the conventionally used Ga FIB systems.

The author acknowledges the facilities at the Central Analytical Research Facility, operated by the Queensland University of Technology 


\section{References}

(1) Mayer, J.; Giannuzzi, L.; Kamino, T.; Michael, J. MRS Bulletin, 2007, 32(5), 400-407

(2) Michael, J. R. Microsc. Microanal., 2006, 12

(3) Traylor, R.; Zhang, R.; Kacher, J.; Douglas, J.O.; Bagot, P.A.J.; Minor, A.M. Acta Materialia, 2020, $184,199-210$ 